\section{Creating Models of C ustom Image Classification Workflows Using Softmax Regression and Support Vector Machine}

\author{
J a son Wolfe ${ }^{1}$, Thomas Bahr ${ }^{2}$ and Xia oying J in ${ }^{1}$ \\ ${ }^{1}$ Ha mis Geospatial Solutions, Inc ., Broomfield, Colora do, USA \\ 2Ha mis Geospatia I Solutions G mbH, Gilc hing, Gemany
}

GI_Forum 2018, Issue 2

Page: 240 - 260

Full Paper

Corresponding Author:

thomas.bahr@hamis.com

DOI: $10.1553 /$ giscience2018_02_s240

\begin{abstract}
This study demonstrates how complex image classification workflows can be built using a visual modelling tool. Models facilitate the comparison of different classifiers while allowing an analyst to experiment with different input features. The models include custom workflow steps for preparing input and training data, training the classifier, classifying images and evaluating the results. The example models presented here were used to classify Sentinel-2 imagery of eastem Texas, USA, into five land-use categories that consisted primarily of vegetation. Separate models were created for Softmax Regression and Support Vector Machine (SVM) classification, each using Sentinel-2 spectral bands and again with an additional entropy texture image as input. The results showed that SVM performed better than Softmax Regression and that the selected texture measure did not improve classification results. A discussion is provided of how the models could be extended further to provide different analysisoptions.
\end{abstract}

\title{
Keywords:
}

c lassific a tion, Softma x Regression, support vector mac hine, model, workflow, trainer

\section{Indroduction}

Image classification is a well-known technique used in remote sensing to map land-cover types and to monitor changes at the Earth's surface over time. Most image-processing software applications include multiple classifiers, along with the ability to display results and to evaluate the accuracy of the classification. Customizing a classification process to go beyond these basic steps typically involves writing an API (Application Programming Interface). Examples of extending a supervised classification workflow include normalizing and randomizing the input data, training the classifier, and minimizing loss prior to classifying images.

This study demonstrates the use of a visual modelling tool in ENVI version 5.5 (available from Harris Geospatial Solutions, Inc.) to build custom Softmax Regression and Support Vector Machine (SVM) classification workflows to perform these steps. Traditionally, these 
classification workflows required users to write APIs. While an API allows more flexibility in customizing workflows, learning the syntax and details of a specific API can be timeconsuming. The benefit of building a model with a visual programming tool is that inputs, outputs, data management operations and processing tasks can be linked with a drag-anddrop user interface instead of learning API code. Models can be packaged and deployed to desktop and cloud-computing environments for reuse and further customization. Model files can also be shared with colleagues and incorporated into larger image-processing models.

As a case study, we compare the results of Softmax Regression and SVM models to classify five vegetation land-cover classes in Sentinel-2 multispectral imagery. We ran each model using 10 spectral bands as input to classification, and again with an additional entropy texture measure for input, to determine whether texture improved classification accuracy. We hypothesize that SVM classification will yield more accurate results than Softmax Regression and that, compared to using the spectral bands alone, adding a texture measure to the spectral bands will improve classification accuracy.

Choosing the right combination of classifiers and selecting appropriate input features are two factors that will affect classification accuracy (Khatami, Mountrakis, \& Stehman, 2016). The next section describes the classifiers and input features used in this study.

\section{Summary of Classifiers}

The Softmax Regression classifier used in ENVI is a form of multinomial logistic regression that can predict the probabilities of the classes based on input features, after weighting them according to their relative importance (Wolfe, Jin, Bahr \& Holzer, 2017). Softmax Regression is most effective with multi-class classification where the classes are mutually exclusive and more than two discrete possible outcomes are possible (Green, 2012). An example is classifying an image into distinct classes such as Cloud, Water, Asphalt and Vegetation. Pal and Foody (2012) found that multinomial logistic regression classification results were similar in accuracy to SVM but required fewer training samples.

SVM is a well-known supervised classifier derived from statistical learning theory. It separates classes using a decision surface that maximizes the margins between the classes (Chang \& Lin, 2001). SVM classification includes several input parameters (Hsu, Chang \& Lin, 2010):

- The kernel type used to give the weights of nearby data points in estimating target classes. Four kernel types are used: Radial Basis Function (RBF), Polynomial, Linear and Sigmoid.

- A penalty value that controls the trade-off between allowing training errors and forcing strict margins.

- Based on the selected kernel type, there are other parameters - for example, the degree of the kernel if the kernel type is Polynomial, the bias if the kernel type is Polynomial or Sigmoid, and the gamma value if the kernel type is RBF, Polynomial or Sigmoid. 
In a scenario where land-cover types with similar spectral characteristics will be separated into discrete classes, SVM is expected to perform better than Softmax Regression. Braun, Weidner and Hinz (2010) discussed how SVM is better suited for use with hyperspectral images or multispectral images that contain many bands. Topaloğlu, Sertel and Musaoglu (2016) compared Maximum Likelihood classification to SVM, for both Landsat 8 and Sentinel-2 imagery. They found that SVM classification of Sentinel-2 data resulted in the highest classification accuracy.

\section{Input Features}

Several studies have demonstrated the effectiveness of Sentinel- 2 spectral bands in land-use classification (Gašparović \& Jogun, 2017; Georgescu, Vaduva \& Datcu, 2017; Sekertekin, Marangoz \& Akein, 2017). Most have found that the Vegetation Red Edge bands are helpful in discriminating between vegetation types (Delegido, Verrelst, Alonso \& Moreno, 2011; Immitzer, Vuolo \& Atzberger, 2016). Qiu, He, Yin and Liao (2017) showed that Vegetation Red Edge bands $5(703.9 \mu \mathrm{m})$ and $6(740 \mu \mathrm{m})$ were the most beneficial in improving the classification of vegetation land-cover classes, particularly agriculture. Rujoiu-Mare, Olariu, Mihai, Nistor and Săvulescu (2017) found that increasing the number of spectral bands as input to image classification improved the separability of land-cover classes.

Additional input features have the potential to increase classification accuracy - for example, height data, spectral indices and texture metrics. Murray, Lucieer and Williams (2010) discuss the importance of taking into consideration spatial information from pixels rather than spectral information alone. Compared to using the spectral bands alone, they achieved higher accuracy by adding grey-level co-occurrence matrix (GLCM) texture metrics as input to classifying six vegetation classes in IKONOS imagery. Similarly, Salas, Boykin and Valdez (2016) improved classification accuracy by using dissimilarity, homogeneity, contrast and second-moment GLCM metrics.

Our study compares the results of image classification using Sentinel-2 spectral bands, with and without an additional first-order entropy texture image. Texture should provide an additional spatial context to image classification, to help distinguish forest and rangeland (rougher textures) from agricultural land (homogenous textures).

\section{Material and Methods}

The case study presented here uses the ENVI Modeler to build two supervised classification workflows: one for Softmax Regression and the other for SVM.

\section{Study Area}

The study area for this research is in eastern Texas, USA, along the Brazos River (Figure 1). The site was chosen because it contains a mix of land-cover classes that are predominantly vegetative, including wetlands, forest, agriculture and rangeland. The area of interest is bounded by coordinates $30.96-31.04^{\circ} \mathrm{N}, 96.74-96.84^{\circ} \mathrm{W}$. 


\section{Wolfe et al}

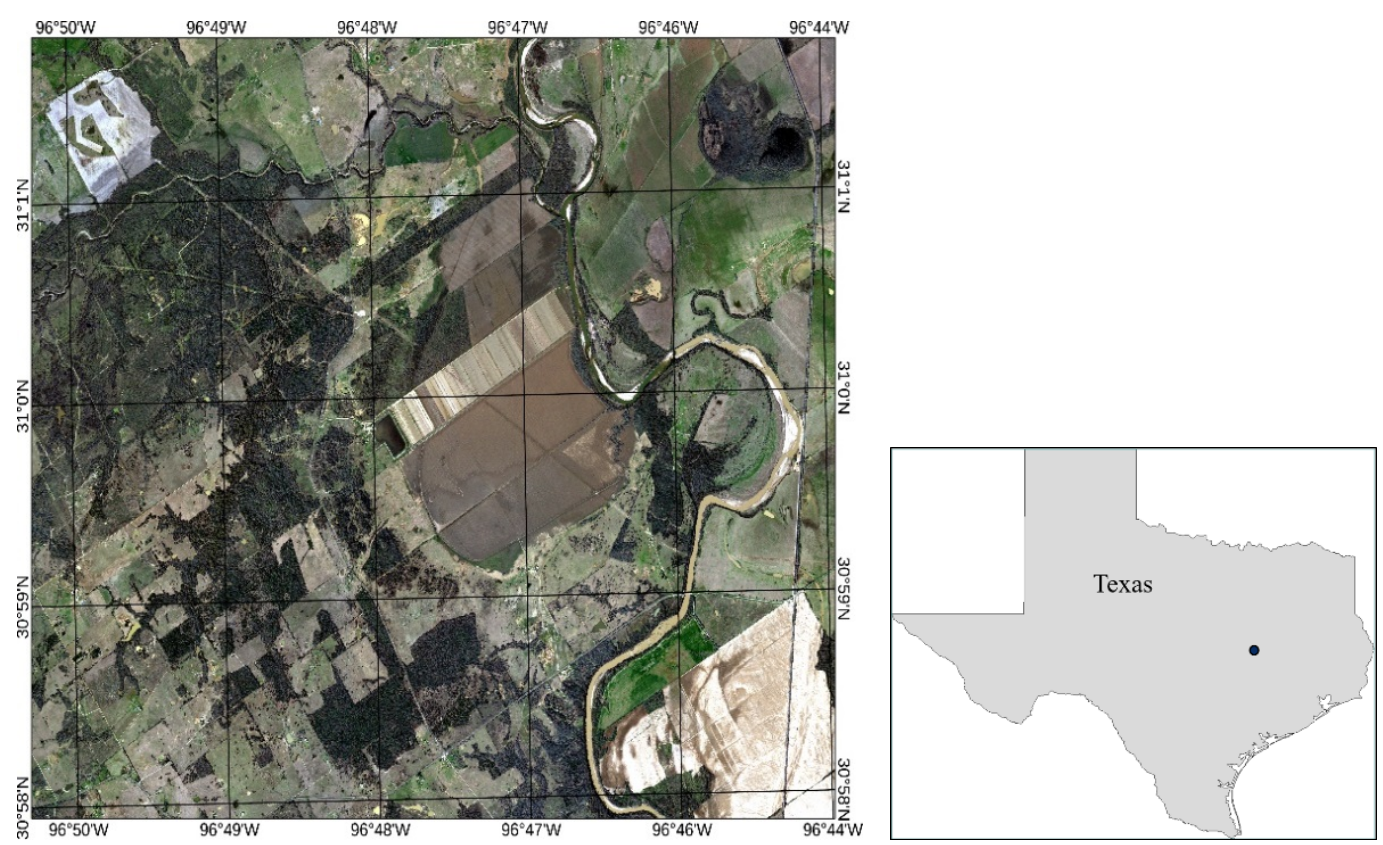

Figure 1: U.S. Geological Survey (USGS) High Resolution Orthophoto mosaic of study a rea (left), overview map (right)

Qiu et al. (2017) similarly chose a study site in eastern Texas for testing Sentinel-2 image classification because of its diverse land-cover types.

\section{Data Preparation}

A Sentinel-2A Level-1C image of the study area was downloaded from the USGS EarthExplorer website (http://earthexplorer.usgs.gov/). The image was acquired on 8 August 2017. Level-1C images have been calibrated to top-of-atmosphere reflectance (ESA, 2015).

Additional processing steps were taken to prepare the image for analysis:

1. Creation of a layer stack of the visible and near-infrared bands, while resampling the 20-metre bands to 10 metres.

2. Definition of a spatial subset around a specific area of interest.

3. Use of QUick Atmospheric Correction (QUAC) to correct the image for atmospheric effects. Pixels represent surface reflectance.

4. Creation and application of a mask of all non-vegetation features such as roads, water and buildings.

5. Updating band names. 
The resulting dataset contained 10 bands (Figure 2):
B2 Blue $(496.6 \mu \mathrm{m})$
B6 Vegetation Red Edge $(740.2 \mu \mathrm{m})$
B3 Green $(560.0 \mu \mathrm{m})$
B7 Vegetation Red Edge $(782.5 \mu \mathrm{m})$
B4 Red $(664.5 \mu \mathrm{m})$
B8A Narrow NIR $(864.8 \mu \mathrm{m})$
B8 NIR $(835.1 \mu \mathrm{m})$
B11 SWIR $(1613.7 \mu \mathrm{m})$
B5 Vegetation Red Edge $(703.9 \mu \mathrm{m})$
B12 SWIR $(2202.4 \mu \mathrm{m})$

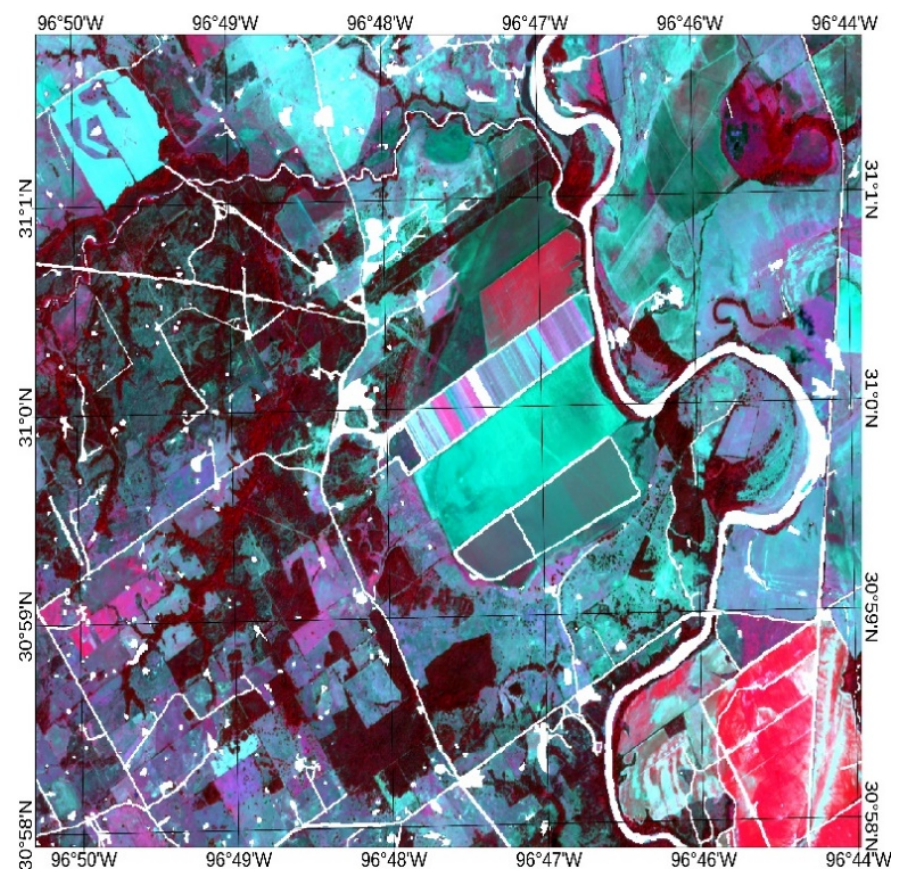

Figure 2: Colour-infrared view of the Sentinel-2 image. White a reas indic ate non-vegetation pixels that were masked out

A first-order entropy texture image was created from the red band (band 4) of the Sentinel-2 image. ENVI uses the technique described in Anys, Bannari, He and Morin (1994) to calculate entropy. Figure 3 shows the resulting entropy image. 


\section{Wolfe et al}

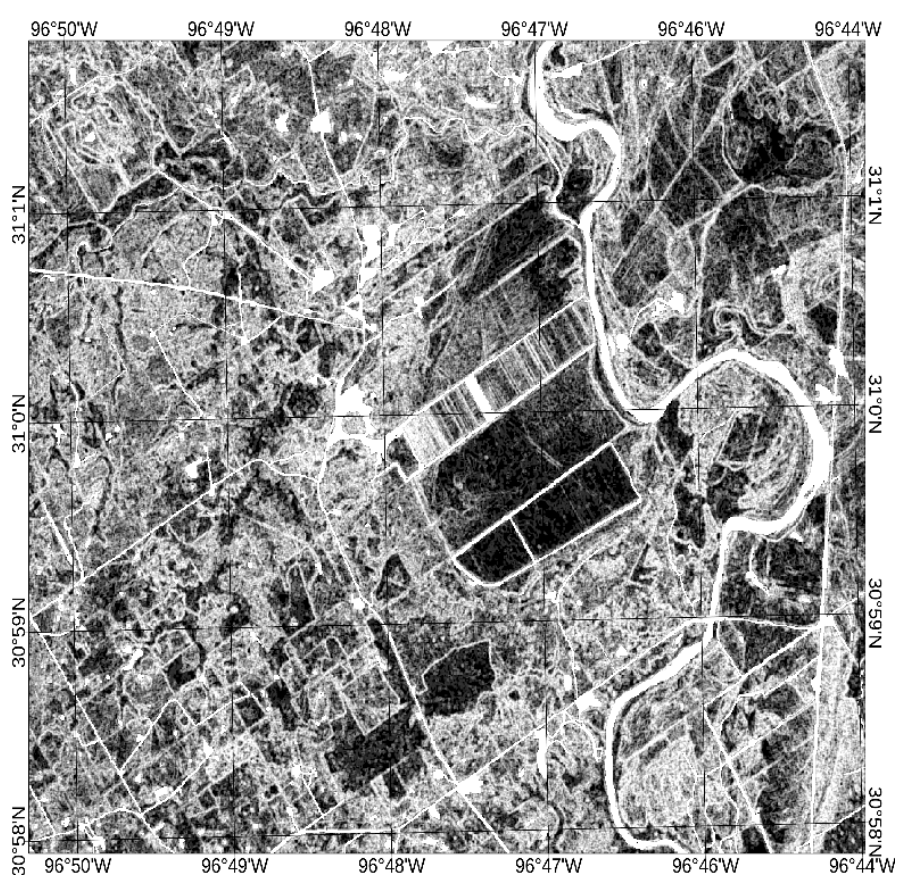

Figure 3: First-order entropy texture image derived from Sentinel-2 Band 4 (664.5 $\mu \mathrm{m})$. Dark areas indicate homogeneous textures that typically correspond to agricultural fields and pastures. Lighter a reas indicate rough textures that typically comespond to forest

A separate layer-stacked image was created using the 10 Sentinel-2 bands and the first-order entropy texture.

\section{Training Data}

Field studies to validate ground truth were not feasible in this study, so training data consisted of samples of Sentinel-2 image pixels that belonged to each feature type. The following feature types were selected for image classification, using land-use terminology from Anderson, Hardy, Roach and Witmer (1976):

- Nonforested Wetland: Characterized by low topography where the water table is above or approaching the surface. In the study area, wetlands are located near river flood plains and contain an abundance of hydrophytic vegetation and wet soils. The Sentinel-2 SWIR bands can help separate these pixels from other classes, since they contain more soil moisture.

- Rangeland: Land dominated by natural vegetation such as grasses, shrubs and brush; often associated with lower moisture levels in the soil and canopy, and sparse vegetation.

- Forest: Areas that contain evergreen and deciduous trees with thick canopies.

- Grass/pasture: Agricultural land used exclusively for growing pasture crops such as alfalfa, or open grassland used for grazing. 


\section{Wolfe et al}

- Cropland: Agricultural land that contains growing or harvested crops, or idle cropland where no planting has occurred. This land-use category is often defined by geometric fields and rows, along with visible tracks where machinery was used for seeding and harvesting. It often has natural and man-made boundaries, along with relatively homogeneous textures.

Distinguishing between these land-use types using remote sensing imagery can be challenging, especially since many areas contain a mix of land-use types. Seldom are the categories defined by abrupt boundaries, except possibly for cropland. Land-use data from the U.S. Department of Agriculture (USDA) CropScape website were used as a rough guide in selecting training samples for each feature type. These data define the approximate locations of primary land-use types such as agriculture, forest, shrubland and grassland/pasture (Figure 4). The class data are from 2017, the same year as the Sentinel-2 image.

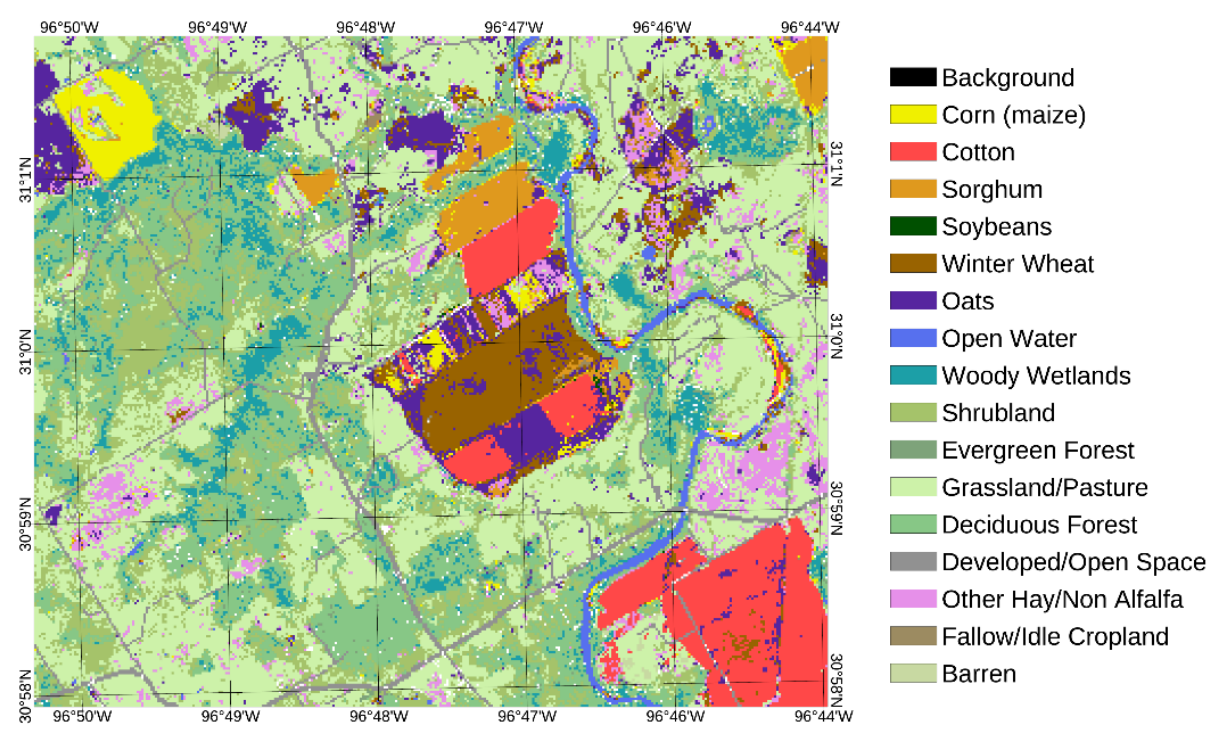

Figure 4: Map of USDA Cropland Data Layer (CDL) classes from 2017 at 30-metre spatial resolution 


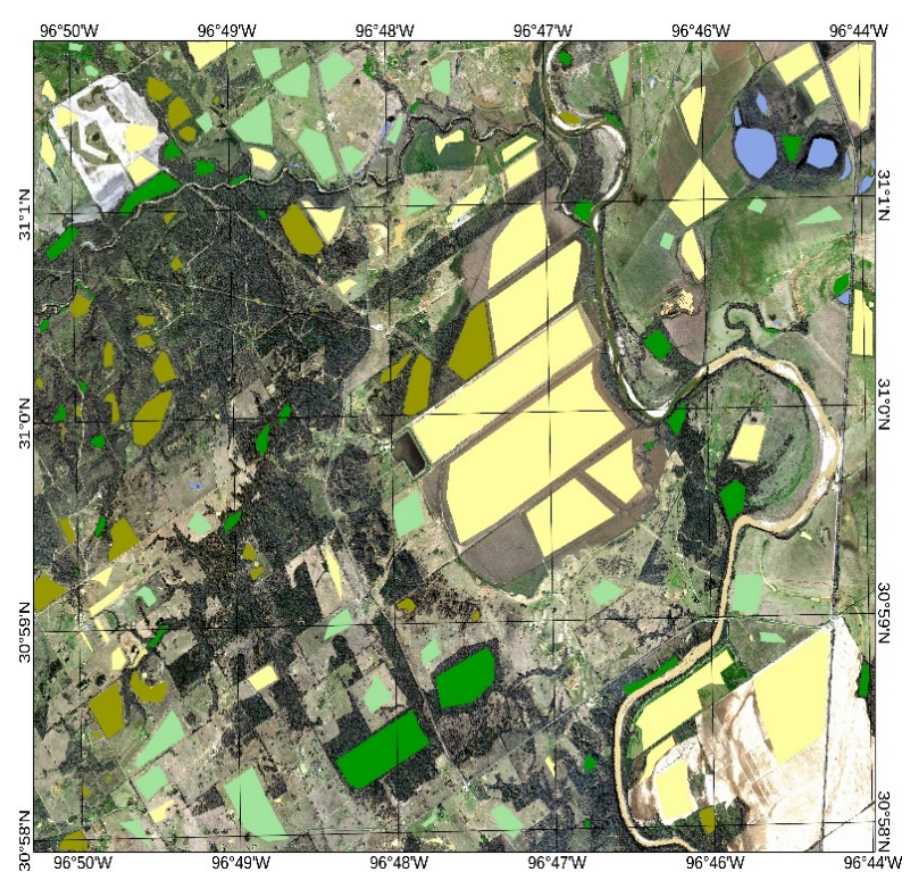

Figure 5: USGS High Resolution Orthophoto mosaic of study area with Regions of Interest training samples (pale yellow $=$ cropland, light green=grass/pasture, dark green=forest, kha ki=rangeland, bluish purple=nonforested wetland)

The image resolution is 10 metres, which makes visual identification of different land-use types difficult. A digital orthophoto of the study area was used as a reference for collecting training samples. The orthophoto is a mosaic of USGS High Resolution Orthophoto images downloaded from the National Map Viewer at https://viewer.nationalmap.gov/basic/. The orthophoto was co-registered with the Sentinel-2 image. It was acquired in 2014, three years prior to the Sentinel-2 image; however, the general land-use types did not change dramatically over a period of three years.

Regions of interest (ROIs) were drawn on the Sentinel-2 image for the five feature types used for classification (Figure 5).

Once the layer-stacked images and training data ROIs were assembled, the classification workflows could be prepared.

\section{Classification Workflows}

Figure 6 shows the typical workflows for Softmax Regression and SVM classification, respectively. Each step in the workflow represents a corresponding ENVI task that contains input and output properties that can be passed from one task to another. 

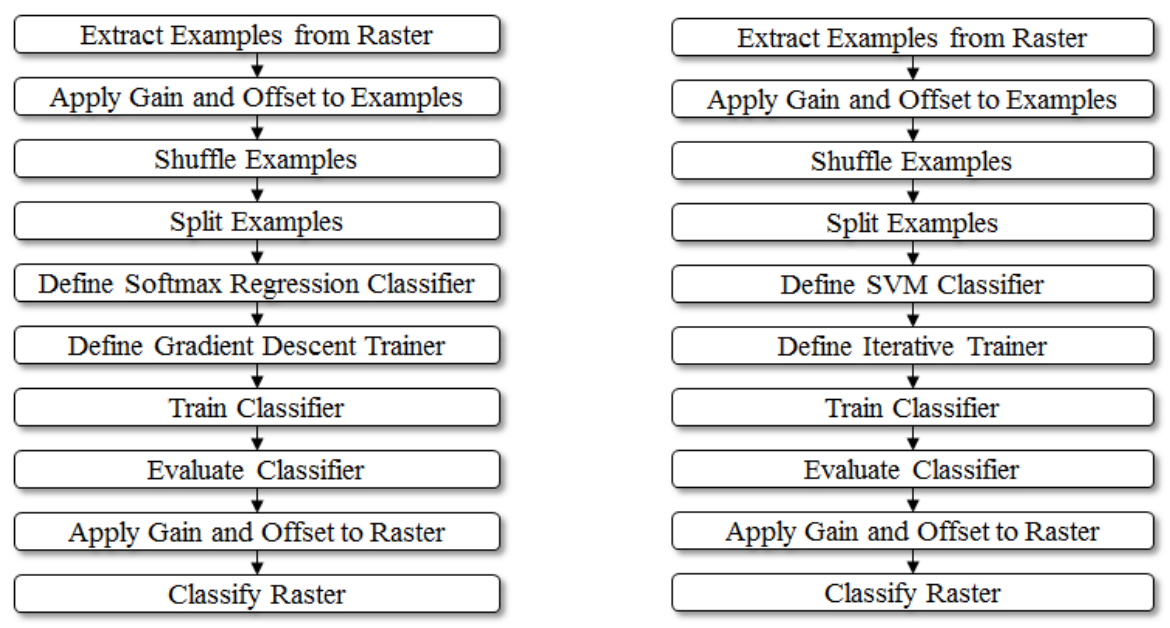

Figure 6: Softmax Regression workflow (left) and SVM classific ation workflow (right) - modified from Wolfe et al., 2017

\section{Preparing the Data}

The first step is to extract examples from the input image. An example is a one-dimensional array of values for a given pixel in all the input bands. This process is automated for every pixel in the image. Class values are also extracted from the input data; these are integers that identify each class.

Classifiers are often sensitive to data values that vary widely (HGS, 2018a). Gains and offsets were applied to the examples to normalize them to a consistent range of values. The normalized examples are shuffled to create a random distribution of data. Finally, the examples are split into two separate arrays. Eighty percent of the examples were used to train the classifier, and the remaining twenty percent were used to validate the classifier.

\section{Training the Classifier}

The role of a trainer is to iteratively minimize the classification error by adjusting the classifier's internal parameters until the loss function converges on a minimum value. This step, which is described in detail by Wolfe et al. (2017) and HGS (2018a), defines the trainer that will be used.

A gradient descent trainer is typically used with a Softmax Regression classifier (Figure 6). This type of trainer iteratively updates the classifier according to the gradient for that iteration. It iterates until the loss converges on a minimum value. The gradient descent trainer requires three inputs:

- Maximum iterations: The maximum number of iterations for which to compute the minimum, if the convergence criterion is not met.

- Convergence criterion: Iterations stop when the change in loss value from the previous iteration falls below a specified threshold. This threshold is the convergence criterion.

- Learning rate: The step size to take downhill during each iteration. 
An iterative trainer is typically used with classifiers such as SVM (Figure 6) that already know how to update themselves without requiring input on how to change.

To train the classifier, one set of examples that were split in the Split Examples task (see Section "Preparing the Data") are passed to the trainer.

\section{Evaluating the Classifier}

This step evaluates the performance of the classifier using the remaining examples (from the Split Examples step) for evaluation. The predicted class values are calculated, and a confusion matrix and accuracy metrics are produced.

\section{Running the Classifier}

The final step is to classify the image. Prior to this step, the gains and offsets computed in the Apply Gains and Offsets to Examples step are applied to the image. According to Wolfe et al. (2017), this ensures that the pixel values are properly scaled among the different feature bands.

\section{Building the Models}

We used the ENVI Modeler to build two models: one for the Softmax Regression classification workflow, and the other for the SVM classification workflow. The models consist of yellow-coloured nodes that represent individual tasks as well as operations that act upon the data (such as extracting array elements, properties and metadata). Nodes can be selected from a list of available tasks and data-processing operations, then dragged and dropped into a canvas to build a model. Nodes are connected so that input and output parameters can be passed from one task to another.

The first part of a classification model (Figure 7) contains steps to prepare the input data for training and classification, as described in Section "Preparing the Data". The Input Parameters node displays a dialogue for the user to select an input image and training data ROIs, and to define the names and locations of output files.

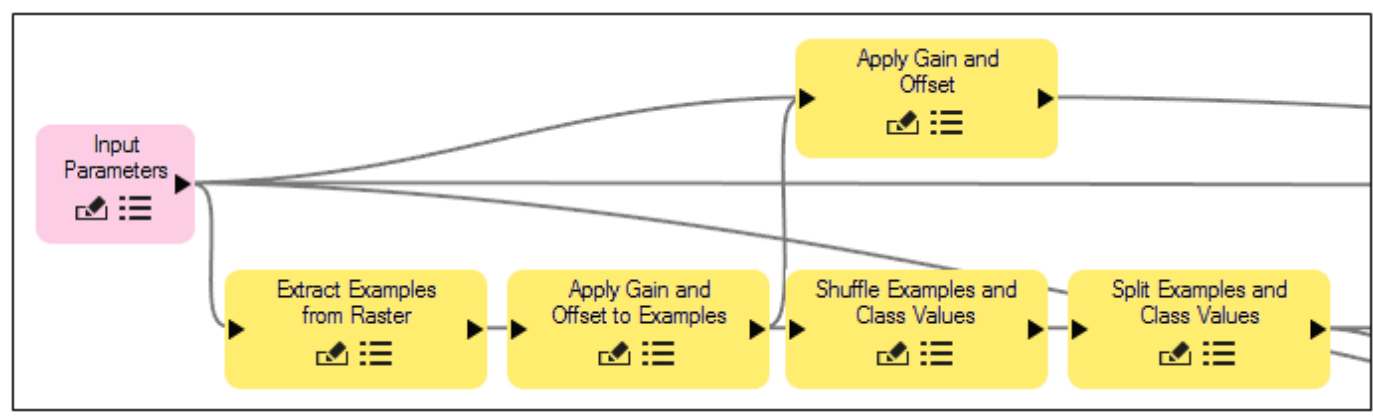

Figure 7: Nodes for preparing input data in the classific ation models 


\section{Wolfe et al}

The next part of the model completes the steps for defining and training the classifier, as described in Section "Training the Classifier". Figure 8 shows an example using the gradient descent trainer for Softmax Regression.

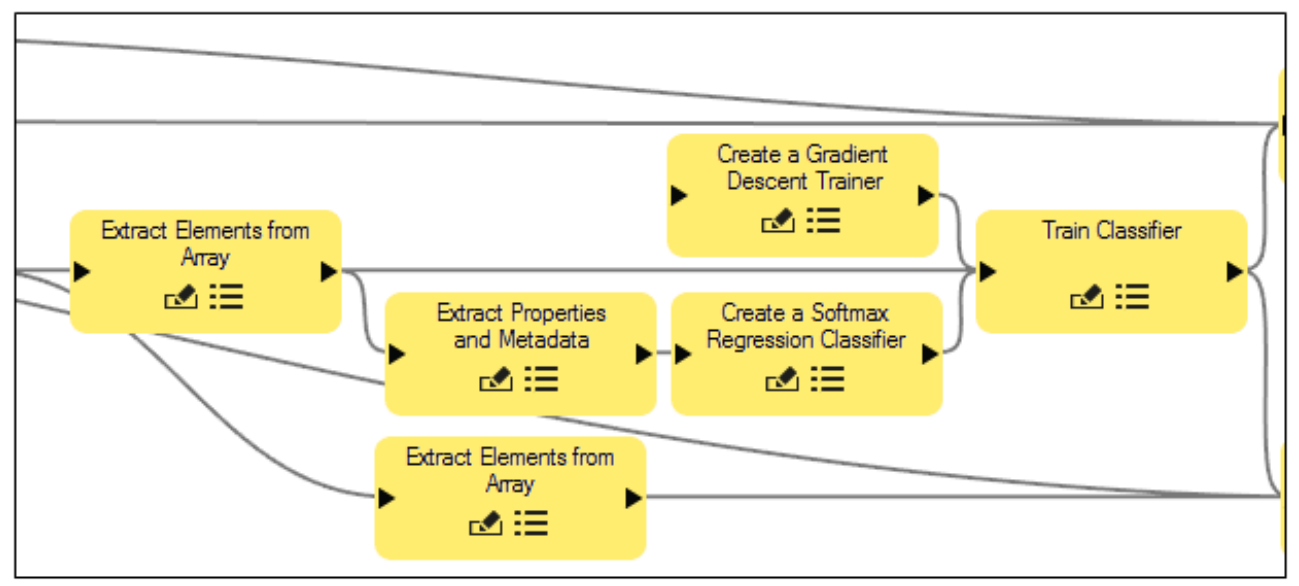

Figure 8: Nodes for defining the trainer and classifier

The examples are split and passed to two different nodes, Train Classifier (Figure 8) and Evaluate Classifier (Figure 10). Because the examples are in array form, they must first be extracted using an Extract Elements from Array node.

Specific metadata values from the examples are required for the Define Softmax Regression Classifier or Define SVM Classifier node, including the class names, number of classes and number of bands. An Extract Properties and Metadata node is used for this purpose. When connecting these two nodes, a connection dialogue appears (Figure 9) so that metadata from the examples can be linked to input parameters required by the classifier nodes.

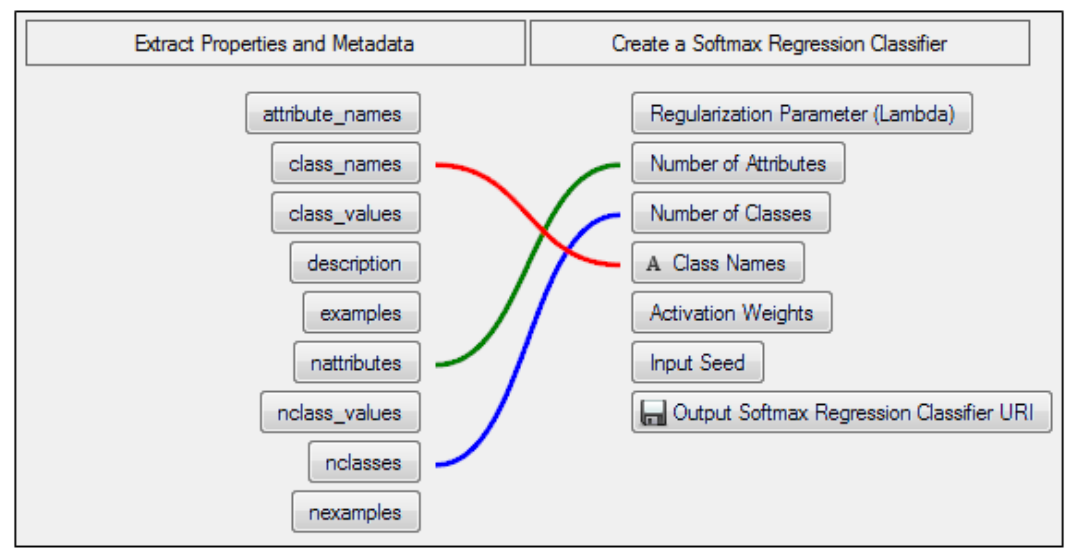

Figure 9: Connecting metadata fields from the examples to input parameters required for the Softmax Regression classifier 


\section{Wolfe et al}

The Create a Gradient Descent Trainer node in the Softmax Regression model contains the input parameters needed for the gradient descent trainer. These must be determined a priori through API tests and plots before creating a model. The following values were used:

- Convergence Criterion: 0.0001

- Maximum Number of Iterations: 800

- Learning Rate: 60

The Create an Iterative Trainer node in the SVM model contains the input parameters needed for the iterative trainer. The following values were used:

- Kernel Type: Radial Basis Function

- Kernel Degree: 2

- Kernel Bias: 1

- Kernel Gamma: Not set

- Penalty: 100

Figure 10 shows the last remaining nodes in the model. The Classify Raster node performs the actual classification. This node requires input from other nodes such as Input Parameters, Apply Gain and Offset and Train Classifier. The Evaluate Classifier node evaluates the trained classifier.

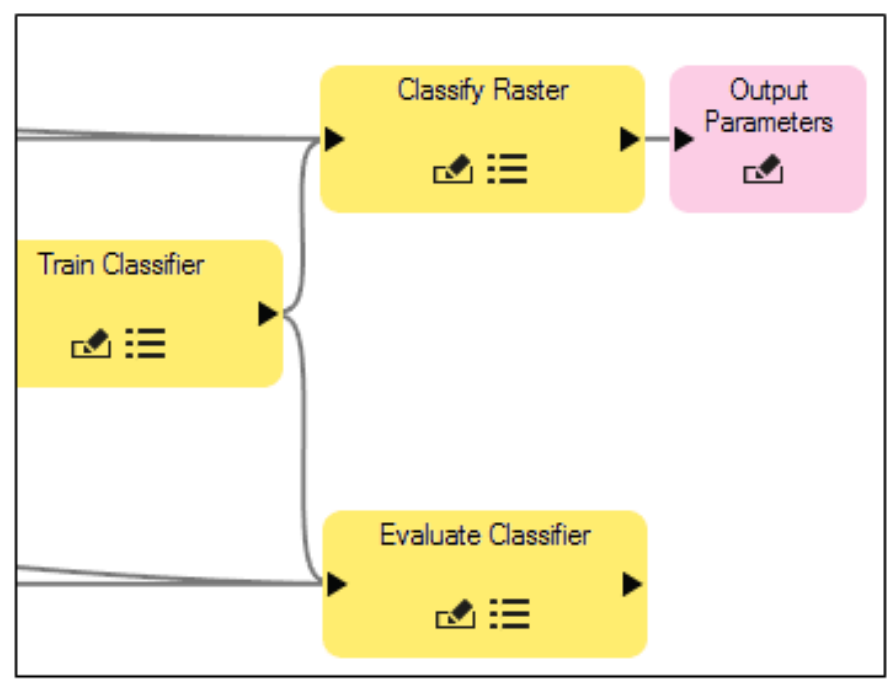

Figure 10: Nodes for classifying the raster and evaluating the classifier

When the model runs, it writes the classification image and confusion matrix to disk. Figure 11 shows a graphical representation of an entire model. 


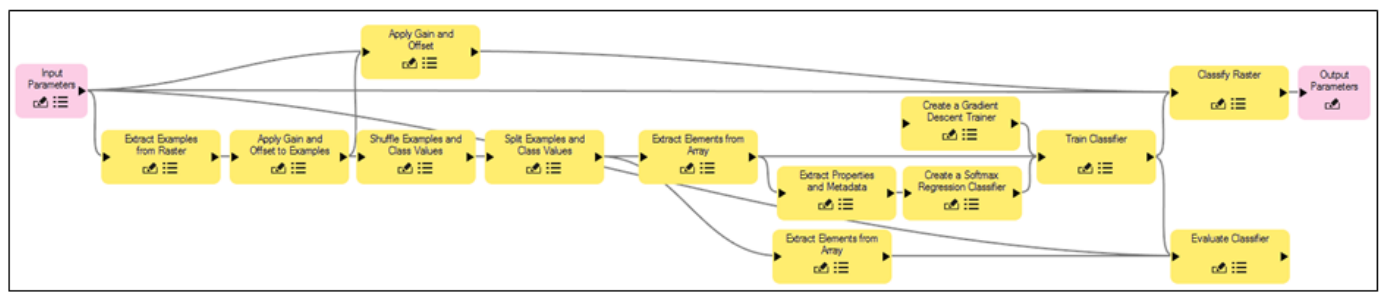

Figure 11: Full Softmax Regression classific ation model

An IDL program was created to restore and print the confusion matrix and accuracy metrics that were saved to disk.

\section{Running the models}

The classification models were run with the following scenarios:

- Softmax Regression using Sentinel-2 bands

- Softmax Regression using Sentinel-2 bands + entropy texture from Band 4

- SVM using Sentinel-2 bands

- SVM using Sentinel-2 bands + entropy texture from Band 4

\section{Results and Discussion}

Models of classification workflows provided a convenient way to experiment with different classifiers and input datasets.

Figures 12-15 show the resulting classification images, and Tables 1-8 show the resulting confusion matrices and accuracy metrics. In general, SVM classification using Sentinel-2 bands performed the best, with an overall accuracy of $89.6 \%$ (Table 6 ). It performed slightly better than the combination of spectral bands and first-order entropy texture $(89.3 \%$, Table 8). SVM performed better than Softmax Regression (71.0\% with texture band, $72.16 \%$ without), so our initial hypothesis was validated. However, the choice of an entropy texture image as an additional feature layer did not improve the classification in either case.

Softmax Regression was not as effective at discriminating between vegetation land-use types. Figures 12 and 13 show an increased mixture of classes, compared to the SVM classifier results in Figures 14 and 15. However, the Softmax Regression models took less than one minute to complete, while the SVM models took nearly two hours, most of which was spent training the classifier.

In both classifiers, pixels that were classified as Nonforested Wetland were spuriously mixed with other classes. Pixels classified as Rangeland often appeared within Cropland and Grass/Pasture regions, where no dry shrubs were present. Pixels classified as Cropland were often mixed with the Grass/Pasture class. The Sentinel-2 bands effectively separated Forest from Rangeland due to differences in vegetation health and greenness. 
With regard to the contribution of entropy texture to classification, flat and homogeneous areas (such as Grass/Pasture and Cropland) remained relatively unchanged in both classifiers, compared to using the spectral bands alone. Adding a texture metric resulted in more pixels being classified as Rangeland (due to its high tonal variation) in the Softmax and SVM classifiers, but it did not improve the overall classification accuracy in either case.

In general, additional experimentation and research is needed to find other ancillary datasets that can more effectively discriminate between the five land-use cases.

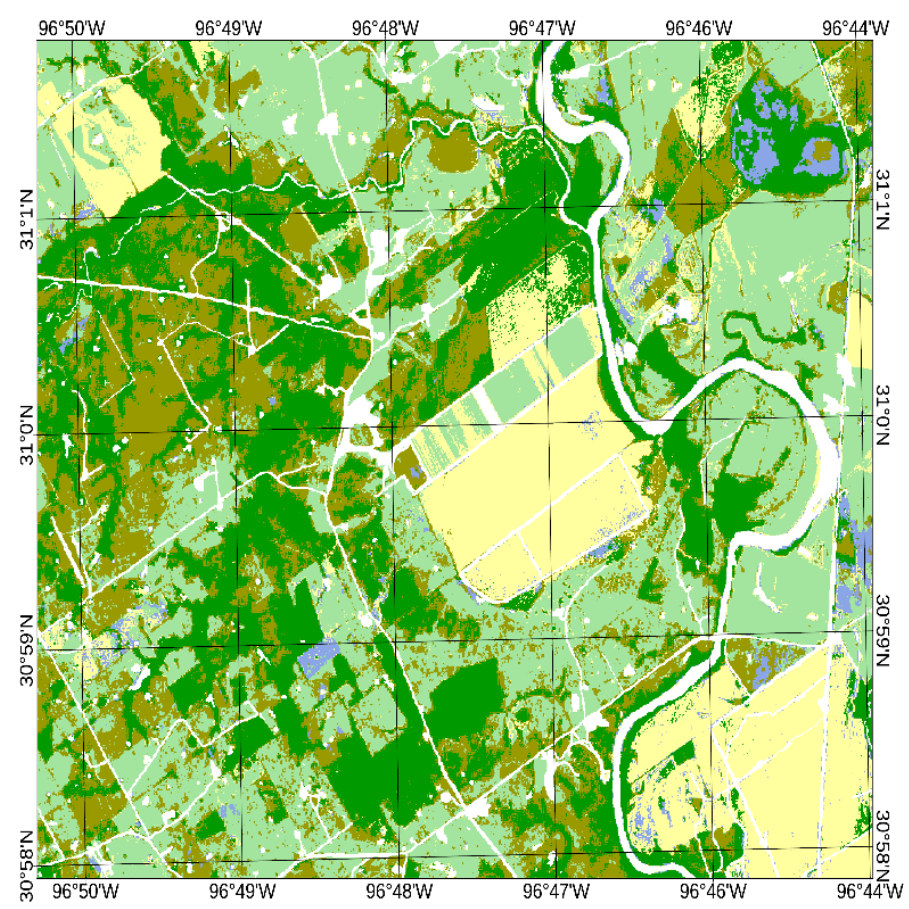

Figure 12: Softmax Regression classific ation image using spectral bands only

Table 1: Confusion matrix from Softmax Regression classific ation using spectral bands only

\begin{tabular}{|c|l|c|c|c|c|c|}
\hline & \multicolumn{7}{|c|}{ Truth } \\
\cline { 2 - 7 } & $\begin{array}{c}\text { Nonforested } \\
\text { Wetland }\end{array}$ & Rangeland & Forest & Grass/Pasture & Cropland \\
\cline { 2 - 7 } & $\begin{array}{l}\text { Nonforested } \\
\text { Wetland }\end{array}$ & 419 & 20 & 5 & 115 & 134 \\
\cline { 2 - 7 }$=$ & 186 & 2689 & 136 & 342 & 1459 \\
\cline { 2 - 7 } & Rangeland & 239 & 567 & 3161 & 8 & 1712 \\
\cline { 2 - 7 } & Forest & 48 & 391 & 12 & 4058 & 2634 \\
\cline { 2 - 7 } & Grass/Pasture & 12 & 10 & 3 & 69 & 10674 \\
\cline { 2 - 7 } & Cropland & & & & & \\
\hline
\end{tabular}




\section{Wolfe et al}

Table 2: Accuracy metric srom Softmax Regression classific ation using spectral bands only

\begin{tabular}{|l|c|c|c|c|c|}
\cline { 2 - 5 } \multicolumn{1}{c|}{} & $\begin{array}{c}\text { Nonforested } \\
\text { Wetland }\end{array}$ & Rangeland & Forest & Grass/Pasture & Cropland \\
\hline $\begin{array}{l}\text { Error of } \\
\text { commission }\end{array}$ & 0.395 & 0.441 & 0.444 & 0.432 & 0.009 \\
\hline $\begin{array}{l}\text { Error of } \\
\text { omission }\end{array}$ & 0.537 & 0.269 & 0.047 & 0.116 & 0.357 \\
\hline F1 value & 0.525 & 0.634 & 0.702 & 0.692 & 0.780 \\
\hline Precision & 0.605 & 0.559 & 0.556 & 0.569 & 0.991 \\
\hline $\begin{array}{l}\text { Producer } \\
\text { accuracy }\end{array}$ & 0.463 & 0.731 & 0.953 & 0.884 & 0.643 \\
\hline Recall & 0.463 & 0.731 & 0.953 & 0.884 & 0.643 \\
\hline $\begin{array}{l}\text { User } \\
\text { accuracy }\end{array}$ & 0.605 & 0.559 & 0.556 & 0.568 & 0.991 \\
\hline
\end{tabular}

\begin{tabular}{|l|l|l|c|}
\hline Overall accuracy & 0.722 & Kappa coefficient & 0.606 \\
\hline
\end{tabular}

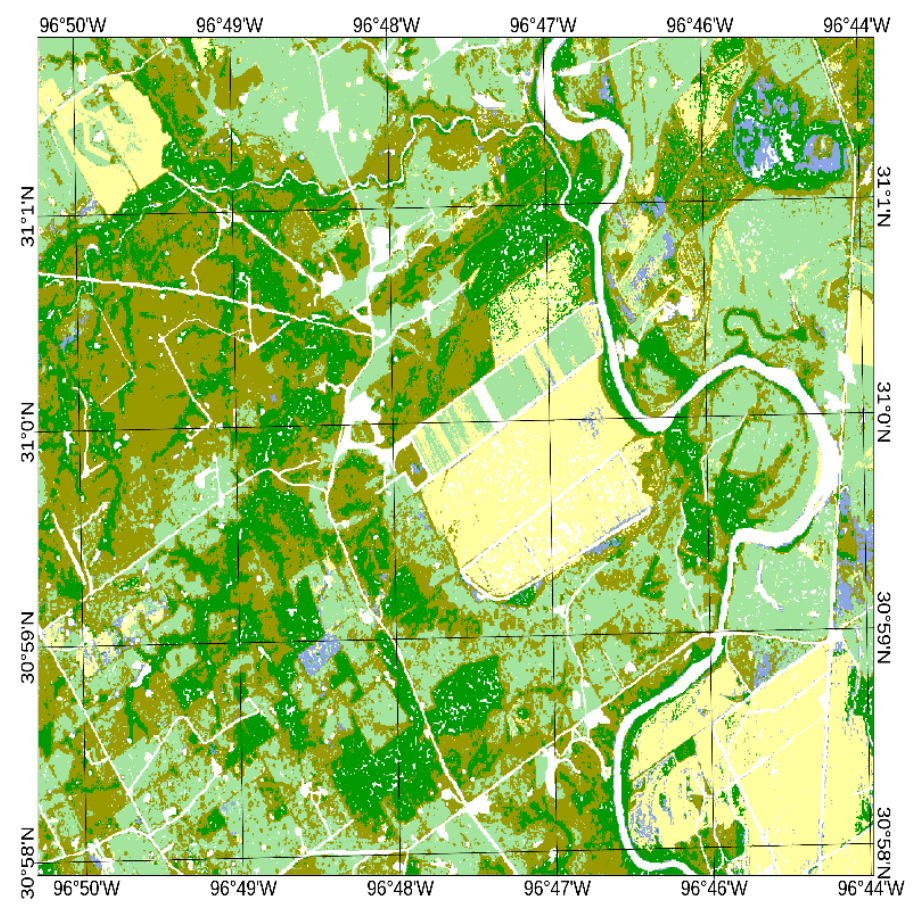

Figure 13: Softmax Regression classific ation image using spectral bands + texture. 


\section{Wolfe et al}

Table 3: Confusion matrix from Softmax Regression cla ssific ation using spectral bands + texture

\begin{tabular}{|c|l|c|c|c|c|c|}
\hline & \multicolumn{5}{|c|}{ Truth } \\
\cline { 2 - 7 } & $\begin{array}{c}\text { Nonforested } \\
\text { Wetland }\end{array}$ & Rangeland & Forest & Grass/Pasture & Cropland \\
\cline { 2 - 7 } & $\begin{array}{l}\text { Nonforested } \\
\text { Wetland }\end{array}$ & 321 & 15 & 7 & 113 & 196 \\
\cline { 2 - 7 }$\approx$ & Rangeland & 176 & 2864 & 204 & 492 & 1424 \\
\cline { 2 - 7 } & Forest & 214 & 412 & 2872 & 4 & 1708 \\
\cline { 2 - 7 } & Grass/Pasture & 22 & 352 & 13 & 3903 & 2595 \\
\cline { 2 - 7 } & Cropland & 3 & 3 & 2 & 62 & 9722 \\
\hline
\end{tabular}

Table 4: Accuracy metric srom Softmax Regression classific a tion using spectral bands + texture

\begin{tabular}{|l|c|c|c|c|c|}
\cline { 2 - 6 } \multicolumn{1}{c|}{} & $\begin{array}{c}\text { Nonforested } \\
\text { Wetland }\end{array}$ & Rangeland & Forest & Grass/Pasture & Cropland \\
\hline $\begin{array}{l}\text { Error of } \\
\text { commission }\end{array}$ & 0.508 & 0.445 & 0.449 & 0.433 & 0.007 \\
\hline $\begin{array}{l}\text { Error of } \\
\text { omission }\end{array}$ & 0.564 & 0.214 & 0.073 & 0.147 & 0.379 \\
\hline F1 value & 0.463 & 0.650 & 0.691 & 0.681 & 0.764 \\
\hline Precision & 0.492 & 0.555 & 0.551 & 0.567 & 0.993 \\
\hline $\begin{array}{l}\text { Producer } \\
\text { accuracy }\end{array}$ & 0.436 & 0.786 & 0.927 & 0.853 & 0.621 \\
\hline Recall & 0.436 & 0.786 & 0.927 & 0.853 & 0.621 \\
\hline $\begin{array}{l}\text { User } \\
\text { accuracy }\end{array}$ & 0.492 & 0.555 & 0.551 & 0.567 & 0.993 \\
\hline
\end{tabular}




\section{Wolfe et al}

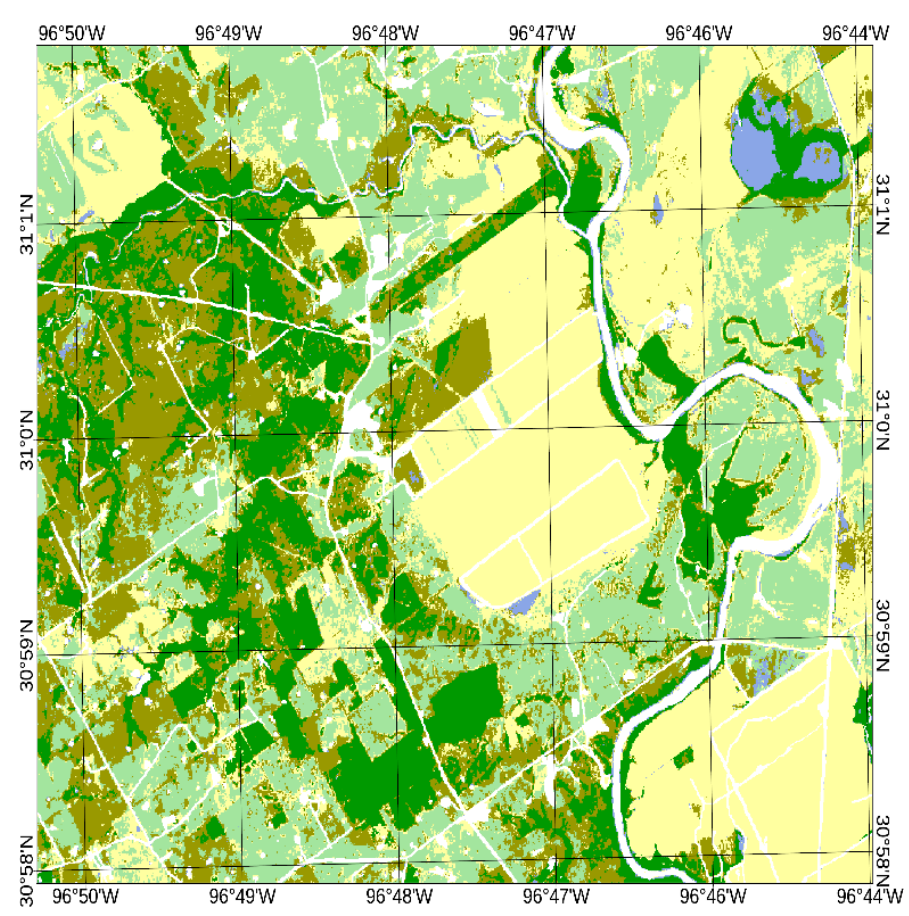

Figure 14: SVM classification image using spectral bands only

Table 5: Confusion matrix from SVM classific a tion using spectral bands only

\begin{tabular}{|c|l|c|c|c|c|c|}
\hline & \multicolumn{7}{|c|}{ Truth } \\
\cline { 3 - 7 } & $\begin{array}{c}\text { Nonforested } \\
\text { Wetland }\end{array}$ & Rangeland & Forest & Grass/Pasture & Cropland \\
\cline { 2 - 7 } & $\begin{array}{l}\text { Nonforested } \\
\text { Wetland }\end{array}$ & 669 & 20 & 11 & 12 & 2 \\
\cline { 2 - 7 } & Rangeland & 100 & 3078 & 115 & 271 & 343 \\
\cline { 2 - 7 } & Forest & 10 & 106 & 3195 & 1 & 0 \\
\cline { 2 - 7 } & Grass/Pasture & 57 & 227 & 15 & 3639 & 870 \\
\cline { 2 - 7 } & Cropland & 56 & 159 & 43 & 613 & 15491 \\
\hline
\end{tabular}




\section{Wolfe et al}

Table 6: Accuracy metrics from SVM classific ation using spectral bands only

\begin{tabular}{|l|c|c|c|c|c|}
\cline { 2 - 6 } \multicolumn{1}{c|}{} & $\begin{array}{c}\text { Nonforested } \\
\text { Wetland }\end{array}$ & Rangeland & Forest & Grass/Pasture & Cropland \\
\hline $\begin{array}{l}\text { Error of } \\
\text { commission }\end{array}$ & 0.063 & 0.212 & 0.035 & 0.243 & 0.053 \\
\hline $\begin{array}{l}\text { Error of } \\
\text { omission }\end{array}$ & 0.250 & 0.143 & 0.054 & 0.198 & 0.073 \\
\hline F1 value & 0.833 & 0.821 & 0.956 & 0.779 & 0.937 \\
\hline Precision & 0.937 & 0.788 & 0.965 & 0.757 & 0.947 \\
\hline $\begin{array}{l}\text { Producer } \\
\text { accuracy }\end{array}$ & 0.750 & 0.857 & 0.946 & 0.802 & 0.927 \\
\hline Recall & 0.750 & 0.857 & 0.946 & 0.802 & 0.927 \\
\hline $\begin{array}{l}\text { User } \\
\text { accuracy }\end{array}$ & 0.937 & 0.788 & 0.965 & 0.757 & 0.947 \\
\hline
\end{tabular}

\begin{tabular}{|l|l|l|c|}
\hline Overall accuracy & 0.896 & Kappa coefficient & 0.832 \\
\hline
\end{tabular}

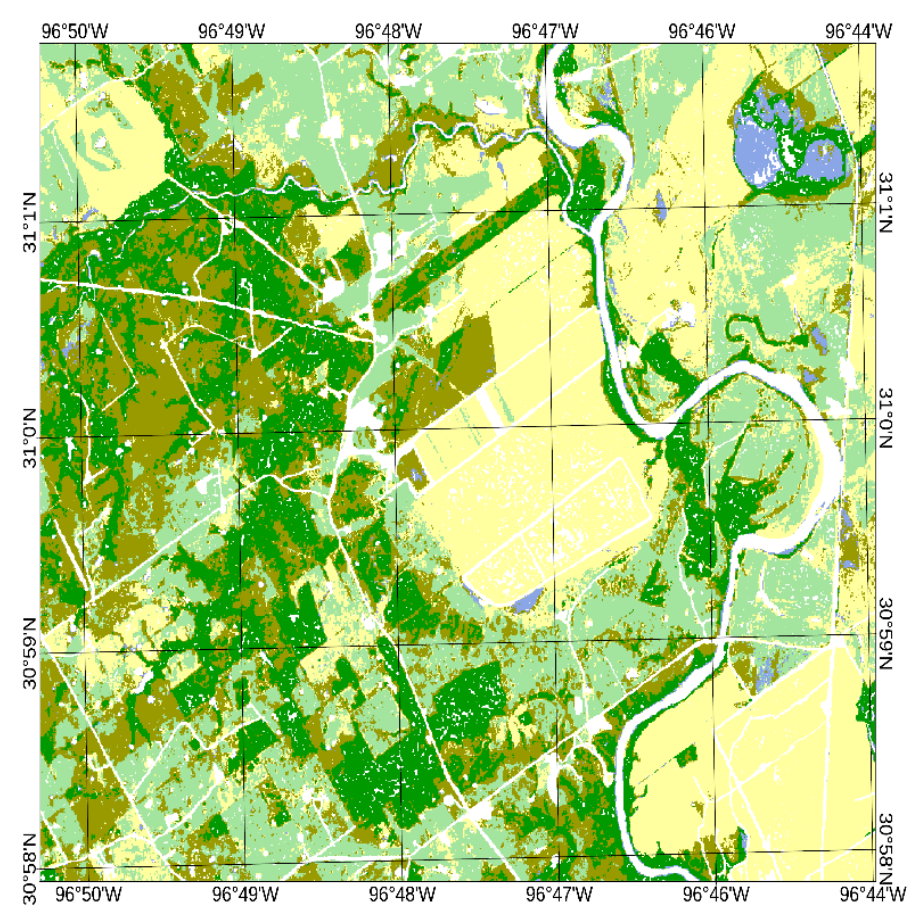

Figure 15: SVM classification image using spectral bands + texture 
Wolfe et al

Table 7: Confusion matrix from Softmax Regression classific ation using spectral bands + texture

\begin{tabular}{|c|l|c|c|c|c|c|}
\hline & \multicolumn{5}{|c|}{ Truth } \\
\cline { 2 - 7 } & $\begin{array}{c}\text { Nonforested } \\
\text { Wetland }\end{array}$ & Rangeland & Forest & Grass/Pasture & Cropland \\
\cline { 2 - 7 } & $\begin{array}{l}\text { Nonforested } \\
\text { Wetland }\end{array}$ & 621 & 23 & 21 & 14 & 0 \\
\cline { 2 - 7 } & Rangeland & 113 & 2991 & 111 & 246 & 233 \\
\cline { 2 - 7 } & Forest & 14 & 133 & 2939 & 2 & 1 \\
\cline { 2 - 7 } & Grass/Pasture & 50 & 254 & 12 & 3554 & 907 \\
\cline { 2 - 7 } & Cropland & 58 & 154 & 13 & 597 & 14638 \\
\hline
\end{tabular}

Table 8: Accuracy metrics from Softmax Regression classific a tion using spectral bands + texture

\begin{tabular}{|l|c|c|c|c|c|}
\cline { 2 - 6 } \multicolumn{1}{c|}{} & $\begin{array}{c}\text { Nonforested } \\
\text { Wetland }\end{array}$ & Rangeland & Forest & Grass/Pasture & Cropland \\
\hline $\begin{array}{l}\text { Error of } \\
\text { commission }\end{array}$ & 0.085 & 0.190 & 0.049 & 0.256 & 0.053 \\
\hline $\begin{array}{l}\text { Error of } \\
\text { omission }\end{array}$ & 0.275 & 0.159 & 0.051 & 0.195 & 0.072 \\
\hline F1 value & 0.809 & 0.825 & 0.950 & 0.773 & 0.937 \\
\hline Precision & 0.915 & 0.810 & 0.951 & 0.744 & 0.947 \\
\hline $\begin{array}{l}\text { Producer } \\
\text { accuracy }\end{array}$ & 0.725 & 0.841 & 0.949 & 0.805 & 0.928 \\
\hline Recall & 0.725 & 0.841 & 0.949 & 0.805 & 0.928 \\
\hline $\begin{array}{l}\text { User } \\
\text { accuracy }\end{array}$ & 0.915 & 0.810 & 0.951 & 0.744 & 0.947 \\
\hline
\end{tabular}

\section{Overall accuracy}

0.893

Kappa coefficient

0.829

\section{Conclusions}

This study has demonstrated how complex image classification workflows can be represented and built as visual models, using a specific case study for illustration. Model nodes can be easily interchanged to accommodate different trainers and classifiers. Unique metadata fields and properties can be extracted and used as input to multiple nodes. Models can save processing results to disk or to the cloud, and they can include steps to view the classification results. This facilitates the comparison of different classifiers on the same dataset. Model files can also be shared among colleagues or incorporated into existing models.

The models used in this study could be further extended as follows: 


\section{Wolfe et al}

- Experiment with additional feature layers to improve classification accuracy, including height, spectral indices and other co-occurrence texture measures.

- Compare classification results from other supervised methods, such as Maximum Likelihood, Mahalanobis Distance and Minimum Distance, that do not require training.

- Run SVM classification on a time-series of images, as suggested by Rujoiu-Mare et al. (2017). This has the potential to improve the accuracy of the classification.

- Create and train the Softmax Regression and SVM classifiers on a reference dataset using spectral and spatial information, then apply them to similar data in different geographic areas (Wolfe et al., 2017).

- Publish the models as single tasks that can be embedded within other, larger, models.

- Deploy the models as tools that can be embedded within ArcGIS® software on the desktop, in the cloud or online.

\section{References}

Anderson, J., Hardy, E., Roach, J., \& Witmer, R. (1976). A land use and land cover classification system for use with remote sensor data. U.S. Geological Survey Professional Paper 964, Washington, D.C.

Anys, H., Bannari, A., He, D., \& Morin, D. (1994). Texture analysis for the mapping of urban areas using airborne MEIS-II images. Proceedings of the First International Airborne Remote Sensing Conference and Exbibition, 3, 231-245.

Braun, A., Weidner, U., \& Hinz, S. (2010). Support vector machines for vegetation classification: a revision. Photogrammetrie Fernerkundung Geoinformation, 4, 273-281.

Chang, C.-C., \& Lin, C.-J. (2001). LIBSVM: A library for support vector machines. Retrieved from https://www.csie.ntu.edu.tw/ cjlin/libsvm/

Delegido, J., Verrelst, J., Alonso, L., \& Moreno, J. (2011). Evaluation of Sentinel-2 red-edge bands for empirical estimation of green LAI and chlorophyll content. Sensors, 11:7, 7063-7081.

European Space Agency (ESA). (2015). Sentinel-2 MSI technical guide, Level-1C algorithm. Retrieved from https://sentinel.esa.int/web/sentinel/technical-guides/sentinel-2-msi/level-1c/algorithm

Gašparović, M., \& Jogun, T. (2017). The effect of fusing Sentinel-2 bands on land-cover classification. International Journal of Remote Sensing, 39:3, 822-841.

Georgescu, F., Vaduva, C., \& Datcu, M. (2017). Land cover classification using Sentinel-2 data. ESA World Cover 2017, Frascati, Italy.

Greene, W. H.. (2012). Econometric Analysis (seventh edition). Boston: Pearson Education.

Harris Geospatial Solutions (HGS) (2018a). ENVI API v. 3.2 documentation. Retrieved from https://www.harrisgeospatial.com/docs/programmingguideclassification_defineclassifier.html

Harris Geospatial Solutions (HGS) (2018b). ENVI API v. 3.2 documentation. Retrieved from https://www.harrisgeospatial.com/docs/programmingguideclassification_codeexamplesoftmaxtas ks.html

Hsu, C.-W., Chang, C.-C., \& Lin, C.-J. (2010). A practical guide to support vector classification. Retrieved from http://ntu.csie.org/ cjlin/papers/guide/guide.pdf

Immitzer, M., Vuolo, F., \& Atzberger, C. (2016). First experience with Sentinel-2 data for crop and tree species classifications in Central Europe. Remote Sensing 8:3, 166.

Khatami, R., Mountrakis, G., \& Stehman, S. (2016). A meta-analysis of remote sensing research on supervised pixel-based land-cover image classification processes: General guidelines for practitioners and future research. Remote Sensing of Environment, 177, 89-100. 


\section{Wolfe et al}

Murray, H., Lucieer, A., and Williams, R. (2010). Texture-based classification of sub-Antarctic vegetation communities on Heard Island. International Journal of Applied Earth Observation and Geoinformation, 12, 138-149.

Pal, M. \& Foody, G. (2012). Evaluation of SVM, RVM and SMLR for accurate image classification with limited ground data. IEEE Journal of Selected Topics in Applied Earth Observations and Remote Sensing, 5:5, 1344-1355.

Patel, D. \& Stonham, T. (1992). Texture image classification and segmentation using RANK-order clustering. $11^{\text {th }}$ LAPR International Conference on Pattern Recognition, 92-95.

Qiu, S., He, B., Yin, C., \& Liao, Z. (2017). Assessments of Sentinel-2 vegetation red-edge spectral bands for improving land cover classification. ISPRS Archives, XLII-2/W7, 871-874. doi:10.5194/isprs-archives-XLII-2-W7-871-2017.

Rujoiu-Mare, M-R., Olariu, B., Mihai, B-A., Nistor, C., \& Săvulescu, I. (2017). Land cover classification in Romanian Carpathians and Subcarpathians using multi-date Sentinel-2 remote sensing imagery. European Journal of Remote Sensing, 50:1, 496-508.

Salas, E., Boykin, K., \& Valdez, R. (2016). Multispectral and texture feature application in imageobject analysis of summer vegetation in Eastern Tajikistan Pamirs. Remote Sensing, 8:78, 1-20.

Sekertekin, A., Marangoz, A., \& Akein, H. (2017). Pixel-based classification analysis of land use land cover using Sentinel-2 and Landsat-8 data. ISPRS Archives, XLII-4/W6, 91-93. doi: 10.5194/isprsarchives-XLII-4-W6-91-2017.

Stanford University. (2013). Unsupervised feature learning and deep learning. Retrieved from http://ufldl.stanford.edu/wiki/index.php/Softmax_Regression

Topaloğlu, R., Sertel, E., \& Musaoglu, N. (2016). Assessment of classification accuracies of Sentinel-2 and Landsat-8 data for land cover / use mapping. ISPRS Archives, XLI-B8, 1055-1059. doi:10.5194/isprsarchives-XLI-B8-1055-2016.

USDA National Agricultural Statistics Service Cropland Data Layer. (2016). Published crop-specific data layer [Online]. Retrieved from https://nassgeodata.gmu.edu/CropScape/

Wolfe, J., Jin, X., Bahr, T., \& Holzer, N. (2017). Application of softmax regression and its validation for spectral-based land cover mapping. ISPRS Archives, XLII-1/W1, 455-459. doi:10.5194/isprsarchives-XLII-1-W1-455-2017.

Wu, T.-F., Lin, C.-J., \& Weng, R. (2004). Probability estimates for multi-class classification by pairwise coupling. Journal of Machine Learning Research, 5, 975-1005. 